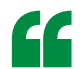

mGAT 1 and mGAT4 inhibitors dramatically reduced the amplitude of optogenetically induced IPSCs

\title{
Midbrain neurons recycle GABA
}

Many dopamine neurons in the substantia nigra pars compacta $(\mathrm{SNc})$ and ventral tegmental area (VTA) signal to striatal projection neurons (SPNs). Mounting evidence indicates that these dopaminergic neurons co-release other neurotransmitters, although the details of this co-transmission remain to be fully established. In a new study, Sabatini and colleagues show that dopaminergic midbrain neurons corelease GABA and that, surprisingly, the source of this neurotransmitter is extracellular.

In a previous study, the authors found that activation of SNc dopamine neurons inhibits SPNs through

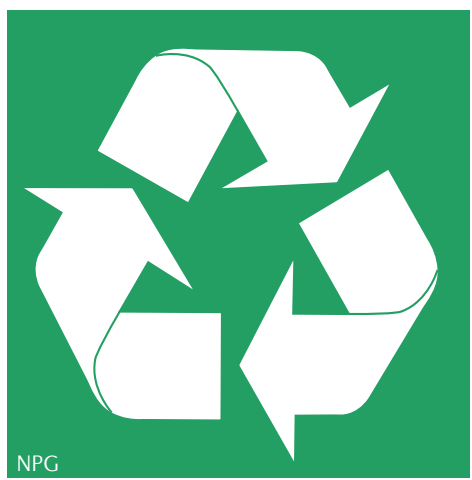

activation of GABA type A (GABA $)$ receptors. Here, the authors showed in ex vivo mouse brain slices that optogenetic activation of VTA neurons evoked inhibitory postsynaptic currents (IPSCs) in SPNs that could be blocked by picrotoxin, a $\mathrm{GABA}_{\mathrm{A}}$ receptor antagonist. Moreover, they found that acute antagonism of the GABA reuptake transporter mGAT1, which is expressed on GABAergic presynaptic terminals, slowed the decay of IPSCs in SPNs after optogenetic activation of SNc or VTA neurons. Together, these results indicate that GABA is co-released by midbrain dopamine neurons.

The authors next sought to determine the origin of the presynaptic GABA using immunohistochemical and in situ hybridization analyses. These approaches revealed that hardly any dopaminergic SNc or VTA neurons exhibited expression of the $65 \mathrm{kDa}$ or $67 \mathrm{kDa}$ isoforms of glutamic acid decarboxylase $\left(\mathrm{GAD}_{65}\right.$ or $\left.\mathrm{GAD}_{67}\right)$ - the main enzymes responsible for GABA synthesis in the CNS - although these neurons did express GABA transaminase, which converts succinate semialdehyde into GABA. However, blockade of GABA transaminase did not reduce optogenetically evoked IPSCs. These data suggest that dopaminergic SNc and VTA neurons do not synthesize GABA for inhibitory transmission.

Strikingly, many SNc and VTA neurons showed expression of mGAT1 and mGAT4, and chronic incubation ( $>30$ minutes) of striatal slices with mGAT1 and mGAT4 inhibitors dramatically reduced the amplitude of optogenetically induced IPSCs in SPNs. Thus, the authors propose that mGAT1 and mGAT4 transporters enable SNc and VTA neurons to 'recycle' extracellular GABA for release.

Overall, this study provides evidence that GABA is co-released by SNc and VTA dopaminergic neurons and that rather than being synthesized de novo, this GABA is derived from outside neurons and taken up by mGATs.

Natasha Bray

ORIGINAL RESEARCH PAPER Tritsch, N. X. et al. Midbrain dopamine neurons sustain inhibitory transmission using plasma membrane uptake of GABA, not synthesis. elife http://dx.doi org/10.7554/eLife.01936 (2014) 